\title{
Eficiencia de aplicación en el riego con surcos cerrados al existir dos pendientes
}

\author{
E. Vázquez-Fernández \\ Instituto de Ingeniería, UNAM \\ Email:ernesfus@servidor.unam.mx
}

(recibido: marzo de 1999; aceptado:mayo de 1999)

\begin{abstract}
Resumen
Se presentan resultados de la eficiencia de aplicación en riego con surcos cerrados y dos pendientes, cuando la mayor se ubica en la cabecera del surco y el cambio de pendiente a la mitad del mismo. En comparación con un surco de pendiente única fuerte y de igual desnivel topográfico en los extremos, el incremento de la eficiencia de aplicación es del orden del $6 \%$. Por tanto, si en el campo se presenta esta situación favorable, no es conveniente nivelar para una sola pendiente a las parcelas, o bien, si ya tienen una sola y fuera posible nivelar el terreno para instalar dos pendientes, la distribución de la lámina de agua sería mejor.
\end{abstract}

Descriptores: surcos cerrados; riego continuo; eficiencia de aplicación.

\begin{abstract}
Results are presented of the application efficiency of closed furrows with two slopes, when the largest is located at the heading of the furrow and the change of slope at mid length. When compared with a furrow with single but pronounced slope and the same topographic drop at each end, the increase of the application efficiency is of about $6 \%$. Therefore, if a favorable situation is encountered at the field, it is not convenient to level up the parcels with a single gradient, or otherwise, if only one slope is available and it becomes possible to level the ground to provide it with two slopes, a better distribution of the water depth could be achieved.
\end{abstract}

Keywords: closed furrows; continuous irrigation; application efficiency.

\section{Introducción}

En el método de riego tradicional con gasto constante (riego continuo) en surcos cerrados se alcanzan eficiencias de aplicación próximas al 90\% (Rendón et al., 1995); sin embargo, cuando las pendientes son fuertes, mayores o iguales de $0.2 \%$, las eficiencias se reducen a valores comprendidos entre el 70 y $80 \%$ (Vázquez y De la Cruz, 1998a). La eficiencia de aplicación para surcos cerrados, si se satisface la dosis o lámina mínima requerida por la planta, equivale a la uniformidad de distribución de las láminas de agua infiltradas, definida como la relación de la lámina acumulada mínima en algún lugar del surco entre la lámina acumulada promedio a lo largo del mismo (Burt et al., 1997).

Este trabajo incluye los resultados de un modelo hidrodinámico completo para riego con surcos cerrados (Vázquez y Gracia, 1998) con dos y una pendiente que reproduce todas las fases del riego (avance, llenado y receso), y que emplea la fórmula de Green y Ampt para calcular el gasto de infiltración vertical a través del perímetro mojado de la sección transversal del surco, un gasto base pequeño $(0.051 / \mathrm{s})$ para determinar las condiciones iniciales de los tirantes y un sólo coeficiente de rugosidad de Manning.

En el diseño de riego por surcos (abiertos o cerrados) la pendiente longitudonal siempre se ha tratado como parámetro y tiene una influencia importante si la pendiente es única (Vázquez, 1996); empero, si el desnivel de una pendiente fuerte (medido desde la cabecera a la parte más baja) es el mismo con dos pendientes diferentes, esta influencia se hace más significativa, repercutiendo en la posibilidad de incrementar la eficiencia de la aplicación si la pendiente mayor se ubica en la cabecera de los surcos.

\section{Procedimiento de cálculo}

El modelo numérico empleado resuelve las ecuaciones completas de Saint-Venant y utiliza, para calcular el gasto 
de infiltración vertical, el perímetro mojado del surco y la fórmula teórica de Green-Ampt (Chu, 1978)

$$
\frac{\mathrm{dZ}}{\mathrm{d} \tau}=\mathrm{K}\left(1+\frac{\lambda}{\mathrm{Z}}\right)
$$

Donde $\mathrm{dZ} / \mathrm{d} \tau$ es la velocidad de infiltración vertical en $\mathrm{cm} / \mathrm{min} ; \tau$ es el tiempo de contacto del agua con el suelo en $\min ; K$ es la conductividad hidráulica en $\mathrm{cm} / \mathrm{min} ; \lambda$ es el producto de las diferencias de humedades y tensiones del suelo en cm y $Z$ es la lámina de infiltración acumulada en $\mathrm{cm}$.

Dado que la lámina de infiltración acumulada se encuentra en el denominador del lado derecho de la ecuación 1 , se requiere un valor inicial diferente de cero; por tanto, en todas las simulaciones numéricas de los dos incisos siguientes y del apéndice, se usa un valor pequeño y constante de $3 \mathrm{~cm}$.

El procedimiento de cálculo seguido para obtener la lámina de infiltración acumulada en el modelo numérico (que usa un esquema en diferencias finitas) es, primero, multiplicar la velocidad de infiltración vertical por el perímetro mojado del surco, $\mathrm{P}$ en $\mathrm{m}$, y la longitud del tramo considerado $\Delta x$ en $\mathrm{m}$, para determinar el gasto de infiltración vertical $\mathrm{Qz}$ en $\mathrm{m}^{3} / \mathrm{s}$. Esto es

$$
\mathrm{Q} z=\left(\frac{1}{6000}\right) \mathrm{P} \Delta x \frac{\mathrm{dZ}}{\mathrm{d} \tau}
$$

Después, al multiplicarse el gasto de infiltración por el intervalo de tiempo $\Delta \mathrm{t}$ en segundos, el volumen de infiltración vertical $\mathrm{Vz}$ en $\mathrm{m}^{3}$, es

$$
\mathrm{Vz}=\mathrm{Q} z \mathrm{t}
$$

Finalmente, si cada volumen infiltrado se acumula desde el primer instante $\mathrm{J}=1$, hasta el último $\mathrm{J}=\mathrm{u}$, durante el tiempo de contacto del agua con el suelo y se divide al final entre un área formada por la misma longitud $\Delta \mathrm{x}$ y la separación entre surcos $\mathrm{D}$ en $\mathrm{m}$, se obtiene la lámina de infiltración acumulada $Z_{i}$ en $\mathrm{m}$, para cada tramo i.

$$
\mathrm{Z}_{\mathrm{i}}=\frac{\sum_{\mathrm{j}=1}^{\mathrm{j}=\mathrm{u}} \mathrm{V} z^{\mathrm{j}}}{\Delta \mathrm{xD}}
$$

Cuando algún valor $Z_{i}$ es igual a la dosis $\mathrm{Zm}$, que deseaba infiltrarse, el riego es satisfactorio.

\section{Simulaciones numéricas en surcos con una pendiente}

Los surcos de sección transversal parabólica empleados en este trabajo fueron tomados de Bautista y Wallender (1993a, surco Schwank1), Vázquez y de la Cruz (1998b, surco Celaya) y Camacho et al. (1997, surco Córdoba), con los parámetros geométricos mostrados en la tabla 1 para calcular el área de la sección transversal A y el radio hidráulico R, según las ecuaciones

$$
\begin{aligned}
& A=\sigma_{1} y^{\sigma 2} \\
& R=\rho_{1} A^{\rho 2}
\end{aligned}
$$

Los parámetros $\sigma 1, \sigma 2, \rho 1, \rho 2$ se obtienen después de tomar logaritmos en ambos lados de las ecuaciones, a través de un ajuste por regresión lineal simple. El tirante del flujo es $\underline{y}$.

Tabla 1. Parámetros de los surcos

\begin{tabular}{lcccc}
\hline \hline $\begin{array}{c}\text { Nombre del } \\
\text { surco }\end{array}$ & $\sigma 1$ & $\sigma 2$ & $\rho 1$ & $\rho 2$ \\
\hline \hline Schwankl (Sc) & 1.0 & 1.696 & 0.333 & 0.508 \\
Celaya (Cy) & 0.913 & 1.5 & 0.43 & 0.555 \\
Córdoba (Cd) & 1.0409 & 1.6383 & 0.4981 & 0.596 \\
\hline \hline
\end{tabular}

Los parámetros de la ecuación de Green-Ampt K y $\lambda$; longitud L; separación entre surcos $\mathrm{D}$; dosis $\mathrm{Zm}$ y desnivel $\Delta z$ se muestran en la tabla 2 de datos principales para diferentes surcos. Estos se identifican en la primera columna por el nombre abreviado de la tabla 1 asociado con el número de simulación entre paréntesis. El coeficiente de rugosidad de Manning no se anota porque se ha utilizado un valor igual a $0.05 \mathrm{~s} / \mathrm{m}^{1 / 3}$ en todos los casos.

En la tabla 3 se muestran los resultados para diferentes valores de la pendiente, $S_{0}$ y gasto $Q$. Ahí ta es la duración de la etapa de avance del agua, $\mathrm{Tr}$, el tiempo de riego y $\eta$, la eficiencia de aplicación. En todos los casos se satisface la dosis y el error en la ecuación de continuidad (volumen de ingreso $=$ volumen infiltrado) es menor de 3\%; además, el tirante máximo del flujo siempre fue menor que la altura de los bordos, por lo que no serían rebasados. La eficiencia de aplicación en \%, se calcula como

$$
\eta=\frac{\mathrm{LDZm}}{6 \mathrm{QTr}}
$$

Donde $L$ está dado en $\mathrm{m}, \mathrm{Z}$ y $\mathrm{D}$ en $\mathrm{cm}$, Tr en min y $\mathrm{Q}$ en $1 / \mathrm{s}$. 
DOI: http://dx.doi.org/10.22201/fi.25940732e.2000.01n3.014

E. Vázquez-Fernández

Tabla 2. Datos principales

\begin{tabular}{lcccccc}
\hline Surco & $\begin{array}{c}\mathrm{K} \\
(\mathrm{cm} / \mathrm{min})\end{array}$ & $\begin{array}{c}\lambda \\
(\mathrm{cm})\end{array}$ & $\begin{array}{c}\mathrm{L} \\
(\mathrm{m})\end{array}$ & $\begin{array}{c}\mathrm{D} \\
(\mathrm{cm})\end{array}$ & $\begin{array}{c}\mathrm{Zm} \\
(\mathrm{cm})\end{array}$ & $\begin{array}{c}\Delta z \\
(\mathrm{~cm})\end{array}$ \\
\hline $\mathrm{Sc}(1)$ & 0.013 & 57.0 & 230 & 75.1 & 18.0 & 46.000 \\
$\mathrm{Cd}(2)$ & 0.017 & 44.0 & 225 & 74.8 & 17.5 & 47.250 \\
$\mathrm{Cy}(3)$ & 0.019 & 40.0 & 220 & 75.0 & 17.0 & 47.850 \\
$\mathrm{Sc}(4)$ & 0.022 & 33.0 & 215 & 75.1 & 16.5 & 48.375 \\
$\mathrm{Cd}(5)$ & 0.024 & 31.0 & 210 & 74.8 & 16.0 & 48.825 \\
$\mathrm{Cy}(6)$ & 0.027 & 29.0 & 205 & 75.0 & 15.5 & 49.200 \\
$\mathrm{Sc}(7)$ & 0.030 & 23.0 & 200 & 75.1 & 15.0 & 49.500 \\
$\mathrm{Cd}(8)$ & 0.032 & 20.7 & 195 & 74.8 & 14.5 & 49.725 \\
$\mathrm{Cy}(9)$ & 0.035 & 20.0 & 190 & 75.0 & 14.0 & 49.875 \\
$\mathrm{Sc}(10)$ & 0.038 & 17.0 & 185 & 75.1 & 13.5 & 49.950 \\
$\mathrm{Cd}(11)$ & 0.040 & 15.0 & 180 & 74.8 & 13.0 & 49.950 \\
$\mathrm{Cy}(12)$ & 0.043 & 14.5 & 175 & 75.0 & 12.5 & 49.875 \\
$\mathrm{Sc}(13)$ & 0.045 & 13.0 & 170 & 75.1 & 12.0 & 49.725 \\
$\mathrm{Cd}(14)$ & 0.048 & 11.0 & 165 & 74.8 & 11.5 & 49.500 \\
$\mathrm{Cy}(15)$ & 0.051 & 10.0 & 160 & 75.0 & 11.0 & 49.200 \\
$\mathrm{Sc}(16)$ & 0.053 & 10.0 & 155 & 75.1 & 10.5 & 48.825 \\
$\mathrm{Cd}(17)$ & 0.056 & 9.5 & 150 & 74.8 & 10.0 & 48.375 \\
$\mathrm{Cy}(18)$ & 0.059 & 10.0 & 145 & 75.0 & 9.5 & 47.850 \\
$\mathrm{Sc}(19)$ & 0.061 & 7.5 & 140 & 75.1 & 9.0 & 47.250 \\
$\mathrm{Cd}(20)$ & 0.064 & 7.5 & 135 & 74.8 & 8.5 & 46.575 \\
$\mathrm{Cy}(21)$ & 0.066 & 9.0 & 130 & 75.0 & 8.0 & 45.825 \\
$\mathrm{Sc}(22)$ & 0.069 & 8.0 & 125 & 75.1 & 7.5 & 45.000 \\
$\mathrm{Cd}(23)$ & 0.072 & 7.0 & 120 & 74.8 & 7.0 & 44.100 \\
$\mathrm{Cy}(24)$ & 0.074 & 7.0 & 115 & 75.0 & 6.5 & 43.125 \\
$\mathrm{Sc}(25)$ & 0.077 & 6.0 & 110 & 75.1 & 6.0 & 42.075 \\
$\mathrm{Cd}(26)$ & 0.080 & 4.8 & 105 & 74.8 & 5.5 & 40.950 \\
$\mathrm{Cy}(27)$ & 0.083 & 6.7 & 100 & 75.0 & 5.0 & 40.000 \\
\hline \hline & & & & & & \\
& & & & & & \\
\hline
\end{tabular}

\section{Simulaciones numéricas en surcos con dos pendientes}

La tabla 4 muestra los resultados de las simulaciones numéricas donde hay un cambio de pendiente a la mitad del surco, cumpliendo siempre con el desnivel $\Delta z$ de la tabla 2 .

La pendiente de aguas arriba, $S_{1}=2 S_{2}$, es mayor y la de aguas abajo, $S_{2}=(2 / 3) S_{0}$, es menor.

Estas son las posiciones y las pendientes para lograr la máxima eficiencia con un gasto que no erosione el suelo en el tramo de mayor pendiente. Booher (1974) recomienda que este gasto en $1 / \mathrm{s}$, no sea mayor que $0.6 / S$, donde $S$ es la pendiente en $\%$. En la penúltima columna se identifica a la eficiencia de aplicación como $\eta_{1}$ para diferenciarla de la anotada en la tabla 3. Obsérvese que $\eta_{1}>\eta$. El ahorro de agua Ag con respecto al
Tabla 3. Resultados de las simulaciones numéricas con una pendiente

\begin{tabular}{lccccc}
\hline \hline Surco & $\begin{array}{c}\mathrm{S}_{0} \\
(\%)\end{array}$ & $\begin{array}{c}\mathrm{Q} \\
(\mathrm{l} / \mathrm{s})\end{array}$ & $\begin{array}{c}\mathrm{ta} \\
(\mathrm{min})\end{array}$ & $\begin{array}{c}\mathrm{Tr}_{\mathrm{r}} \\
(\mathrm{min})\end{array}$ & $\begin{array}{c}\eta \\
(\%)\end{array}$ \\
\hline \hline $\mathrm{Sc}(1)$ & 0.2000 & 0.80 & 308 & 800 & 81 \\
$\mathrm{Cd}(2)$ & 0.2100 & 0.90 & 266 & 685 & 82 \\
$\mathrm{Cy}(3)$ & 0.2175 & 0.90 & 232 & 655 & 79 \\
$\mathrm{Sc}(4)$ & 0.2250 & 0.95 & 194 & 590 & 79 \\
$\mathrm{Cd}(5)$ & 0.2325 & 1.00 & 190 & 535 & 80 \\
$\mathrm{Cy}(6)$ & 0.2400 & 1.00 & 173 & 510 & 79 \\
$\mathrm{Sc}(7)$ & 0.2475 & 1.00 & 144 & 480 & 78 \\
$\mathrm{Cd}(8)$ & 0.2550 & 1.00 & 146 & 455 & 79 \\
$\mathrm{Cy}(9)$ & 0.2625 & 1.00 & 130 & 430 & 78 \\
$\mathrm{Sc}(10)$ & 0.2700 & 1.00 & 114 & 415 & 77 \\
$\mathrm{Cd}(11)$ & 0.2775 & 1.00 & 111 & 385 & 78 \\
$\mathrm{Cy}(12)$ & 0.2850 & 1.00 & 93 & 360 & 77 \\
$\mathrm{Sc}(13)$ & 0.2925 & 1.00 & 81 & 340 & 76 \\
$\mathrm{Cd}(14)$ & 0.3000 & 0.80 & 145 & 378 & 79 \\
$\mathrm{Cy}(15)$ & 0.3075 & 0.90 & 74 & 320 & 76 \\
$\mathrm{Sc}(16)$ & 0.3150 & 0.75 & 123 & 360 & 77 \\
$\mathrm{Cd}(17)$ & 0.3225 & 0.75 & 146 & 330 & 77 \\
$\mathrm{Cy}(18)$ & 0.3300 & 0.75 & 124 & 310 & 76 \\
$\mathrm{Sc}(19)$ & 0.3375 & 0.75 & 75 & 275 & 76 \\
$\mathrm{Cd}(20)$ & 0.3450 & 0.75 & 98 & 250 & 77 \\
$\mathrm{Cy}(21)$ & 0.3525 & 0.75 & 92 & 235 & 76 \\
$\mathrm{Sc}(22)$ & 0.3600 & 0.75 & 74 & 210 & 76 \\
$\mathrm{Cd}(23)$ & 0.3675 & 0.75 & 75 & 180 & 77 \\
$\mathrm{Cy}(24)$ & 0.3750 & 0.75 & 50 & 165 & 76 \\
$\mathrm{Sc}(25)$ & 0.3825 & 0.75 & 35 & 150 & 75 \\
$\mathrm{Cd}(26)$ & 0.3900 & 0.75 & 29 & 125 & 77 \\
$\mathrm{Cy}(27)$ & 0.4000 & 0.75 & 38 & 110 & 76 \\
\hline & & & & & \\
\hline
\end{tabular}

riego con una pendiente puede calcularse con la ecuación

$$
\mathrm{Ag}=\left(1-\frac{\eta}{\eta_{1}}\right) 100
$$

Las eficiencias de los 27 surcos de las tablas 3 y 4 , así como las de otros 40 que se incluyen en el apéndice, que suman un total de 67, se muestran en la figura 1; en el eje horizontal se identifica el desnivel del surco yen el vertical la eficiencia de aplicación. Obsérvese que en todos los casos la eficiencia del riego es mayor en surcos con dos pendientes. Además, hay una tendencia a que las eficiencias disminuyan conforme aumenta el desnivel del surco, es decir, para surcos de la misma longitud se confirma que a mayor pendiente, menor eficiencia (Vázquez y de la Cruz, 1998a), figura 1. 
DOI: http://dx.doi.org/10.22201/fi.25940732e.2000.01n3.014

Tabla 4. Resultados de las simulaciones numéricas con dos pendientes

\begin{tabular}{|c|c|c|c|c|c|c|c|}
\hline Surco & $\begin{array}{c}S_{1} \\
(\%)\end{array}$ & $\begin{array}{l}\mathrm{S}_{2} \\
(\%)\end{array}$ & $\underset{(1 / s)}{Q}$ & $\underset{\substack{\mathrm{ta} \\
(\mathrm{min})}}{ }$ & $\underset{(\min )}{\mathrm{Tr}}$ & $\begin{array}{l}\eta_{1} \\
(\%)\end{array}$ & $\begin{array}{l}\mathrm{Ag} \\
(\%)\end{array}$ \\
\hline $\mathrm{Sc}(1)$ & 0.2667 & 0.1333 & 0.80 & 309 & 765 & 86 & 8.8 \\
\hline $\mathrm{Cd}(2)$ & 0.2800 & 0.1400 & 0.90 & 267 & 625 & 88 & 6.8 \\
\hline Cy (3) & 0.2900 & 0.1450 & 0.90 & 233 & 615 & 84 & 6.0 \\
\hline $\mathrm{Sc}(4)$ & 0.3000 & 0.1500 & 0.95 & 195 & 560 & 84 & 6.0 \\
\hline $\mathrm{Cd}(5)$ & 0.3100 & 0.1550 & 1.00 & 191 & 485 & 87 & 8.0 \\
\hline $\mathrm{Cy}_{\mathrm{y}}(6)$ & 0.3200 & 0.1600 & 1.00 & 174 & 470 & 85 & 7.1 \\
\hline $\mathrm{Sc}(7)$ & 0.3300 & 0.1650 & 1.00 & 145 & 455 & 83 & 6.0 \\
\hline $\mathrm{Cd}(8)$ & 0.3400 & 0.1700 & 1.00 & 147 & 418 & 85 & 7.1 \\
\hline $\mathrm{Cy}(9)$ & 0.3500 & 0.1750 & 1.00 & 131 & 405 & 83 & 6.0 \\
\hline $\mathrm{Sc}(10)$ & 0.3600 & 0.1800 & 1.00 & 115 & 381 & 83 & 7.2 \\
\hline $\mathrm{Cd}(11)$ & 0.3700 & 0.1850 & 1.00 & 112 & 350 & 84 & 7.1 \\
\hline Cy (12) & 0.3800 & 0.1900 & 0.90 & 123 & 363 & 85 & 9.4 \\
\hline Sc (13) & 0.3900 & 0.1950 & 0.90 & 107 & 340 & 84 & 9.5 \\
\hline Cd (14) & 0.4000 & 0.2000 & 0.80 & 145 & 348 & 86 & 8.1 \\
\hline Сy (15) & 0.4100 & 0.2050 & 0.80 & 104 & 330 & 84 & 9.5 \\
\hline Sc (16) & 0.4200 & 0.2100 & 0.75 & 124 & 323 & 85 & 9.4 \\
\hline $\mathrm{Cd}(17)$ & 0.4300 & 0.2150 & 0.75 & 146 & 303 & 84 & 8.3 \\
\hline Cy (18) & 0.4400 & 0.2200 & 0.75 & 124 & 282 & 83 & 8.4 \\
\hline Sc (19) & 0.4500 & 0.2250 & 0.75 & 76 & 250 & 85 & 10.6 \\
\hline $\mathrm{Cd}(20)$ & 0.4600 & 0.2300 & 0.75 & 98 & 227 & 86 & 10.5 \\
\hline Cy (21) & 0.4700 & 0.2350 & 0.75 & 92 & 213 & 83 & 8.4 \\
\hline $\mathrm{Sc}(22)$ & 0.4800 & 0.2400 & 0.75 & 74 & 185 & 85 & 10.6 \\
\hline $\mathrm{Cd}(23)$ & 0.4900 & 0.2450 & 0.75 & 76 & 168 & 84 & 8.3 \\
\hline Cy (24) & 0.5000 & 0.2500 & 0.75 & 50 & 150 & 84 & 9.5 \\
\hline Sc (25) & 0.5100 & 0.2550 & 0.75 & 35 & 133 & 83 & 9.6 \\
\hline $\mathrm{Cd}(26)$ & 0.5200 & 0.2600 & 0.75 & 30 & 117 & 83 & 7.2 \\
\hline Сy (27) & 0.5333 & 2.2667 & 0.75 & 39 & 101 & 83 & 8.4 \\
\hline
\end{tabular}

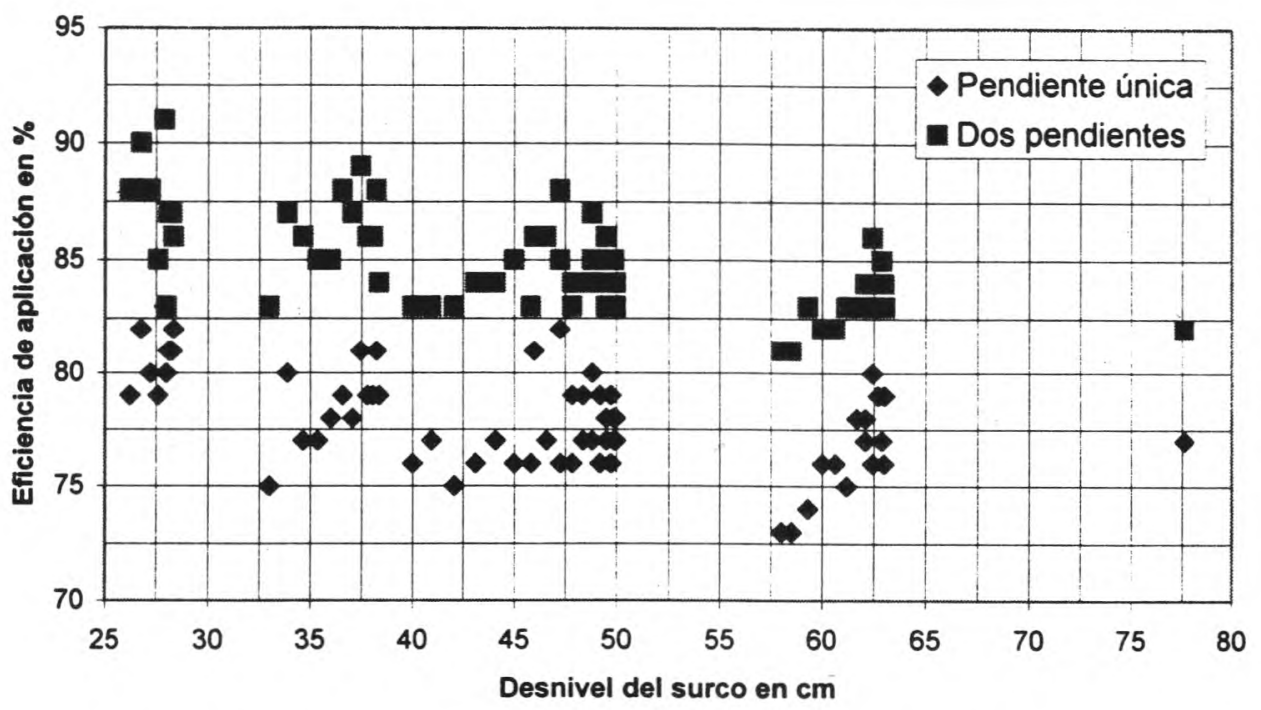

Figura 1. Eficiencia de aplicación según el desnivel del surco 


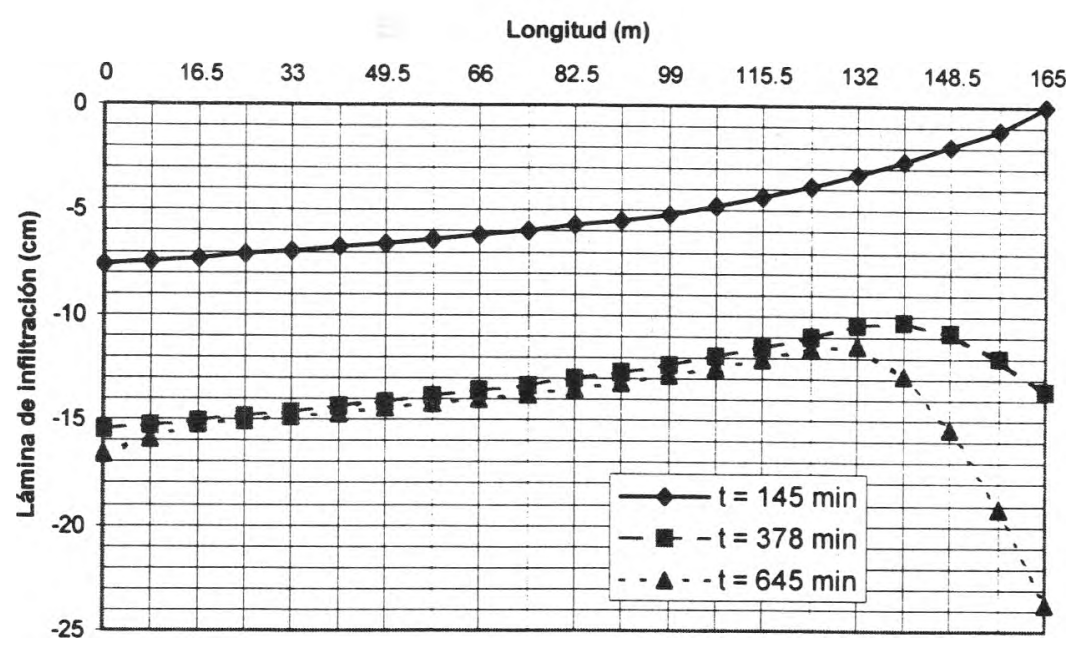

Figura 2. Láminas de infiltración para una pendiente. Surco Cd (14)

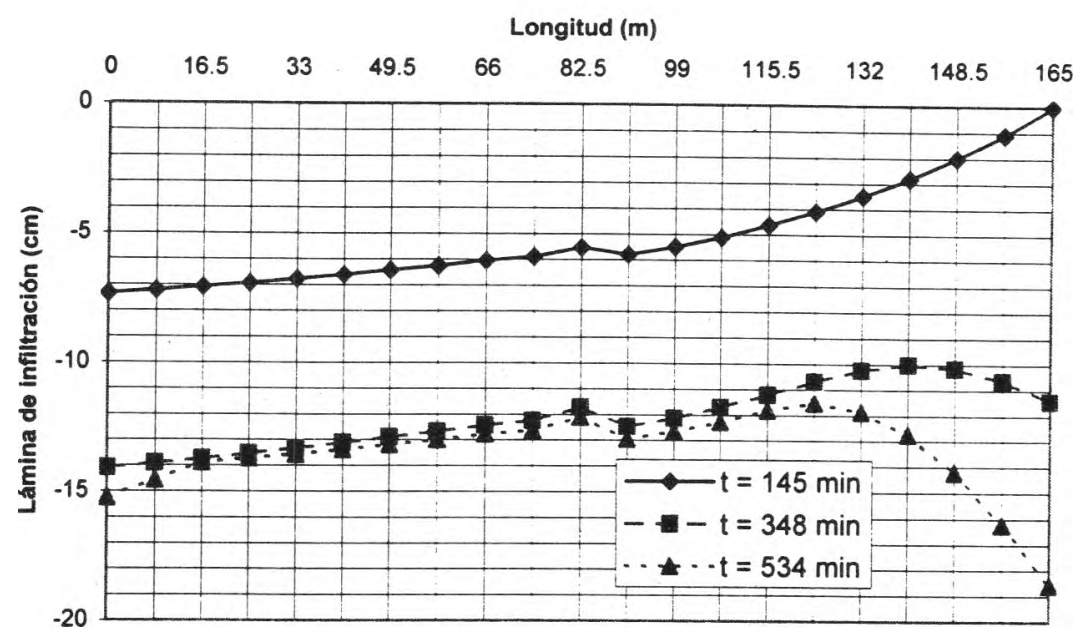

Figura 3. Láminas de infiltración para dos pendientes. Surco Cd (14)

\section{Análisis de resultado}

El incremento de la eficiencia se explica gracias al efecto del remanso producido por la pendiente de menor valor aguas abajo, lo que hace que los perímetros mojados sean mayores hacia el tercio medio del surco, y así se aumente la infiltración y se logre una mejor distribución de la lámina infiltrada. En las figuras 2 y 3 se muestran las láminas de infiltración al final de las fases de avance del agua, llenado y receso para el surco Sc (14) con una y dos pendientes, respectivamente. Véase que para éste último caso (figura 3) las láminas infiltradas son menores en el tercio medio del surco que para una pendiente (figura 2) y que la lámina mínima se alcanza en un sitio más próximo al centro, pero en un tiempo menor.
Desde el punto de vista práctico, la nivelación que requiere dos pendientes en la parcela, con la mayor en la parte alta, produciría un movimiento de tierras que levantaría un volumen de capa vegetal posiblemente perjudicial para el cultivo. Este volumen se calcularía como el producto del área comprendida entre las pendientes $S_{0}, S_{1}$ y $S_{2}$ de la figura 4 , por el ancho de la parcela.

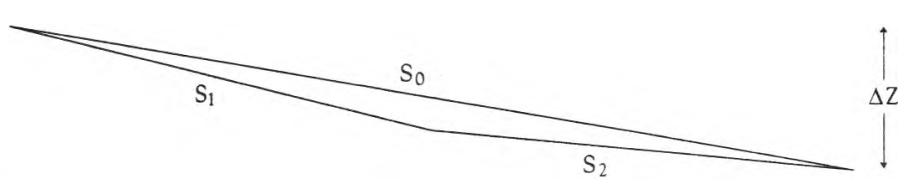

Figura 4. Capa vegetal removida al usar dos pendientes 


\section{Conclusiones}

La modificación de las pendientes en el campo no es práctica común porque se procura nivelar las tierras con la pendiente natural; sin embargo, cuando se tenga una parcela con surcos cerrados y dos pendientes fuertes (mayores o iguales que $0.2 \%$ ) diferentes, situándose la mayor en la parte alta, los resultados de este trabajo permiten concluir que conviene conservarlas.

Ahora bien, si fuera posible cambiar las pendientes de una sola a dos, con la mayor en la parte alta, sin afectar al cultivo por la remoción de la capa vegetal, el ahorro de agua cuando el cambio de pendiente se hace a la mitad del surco, puede llegar a ser hasta de $10 \%$ (ver última columna de la tabla 4).

Es conveniente verificar los resultados teóricos de este trabajo con otro modelo numérico diferente al empleado aquí, pero también debe considerarse la variación del perímetro mojado a lo largo del surco, o bien, preferentemente en el campo.

\section{Agradecimientos}

Al Dr. Jesús Gracia Sánchez por sus opiniones y sugerencias.

\section{Referencias}

Bautista E. y Wallender W. W. (1993a). Reliability of optimized furrow-infiltration parameters. Journal of Irrigation and Drainage Engineering, Vol. 119, No.5.

Booher L. J. (1974). Surface Irrigation. Land and water development series. Food and agriculture organization of the United Nations.

Burt C. M., Clemens A. J., Strelkoff T. S., Solomon K. H., Bliesner R. D., Hardy L. A., Howell T. A. y Eisenhauer D. E. (1997). Irrigation performance measures: efficiency and uniformity. Journal of Irrigation and Drainage Engineering. Vol. 123, No. 6.

Camacho E., Pérez C., Roldán J., Alcaide M., (1997). Modelo de manejo y control en tiempo real del riego por surcos. Ingeniería del Agua, Universidad Politécnica de Valencia, Vol. 4, No. 4.

Chu S. T. (1978). Infiltration during and unsteady rain. Water Resources Research, Vol. 14, No. 3.

Rendón P. L., Fuentes R. C. y Magaña S. G. (1995). Diseño simplificado del riego por gravedad. Memorias del VI Congreso Nacional de Irrigación, Chapingo, México.

Vázquez E.(1996). Formulación de un criterio para incrementar la eficiencia del riego por surcos, incluyendo el método de corte posterior. Tesis de doctorado. División de Estudios de Posgrado de la Facultad de Ingeniería, UNAM, Ciudad Universitaria, México.

Vázquez E. y Gracia J. (1998). Calibración de parámetros de infiltración y rugosidad con un modelo numérico para riego con surcos cerrados. Ingeniería del Agua, Universidad Politécnica de Valencia, España (en prensa).

Vázquez E. y De la Cruz R. (1998a). Criterio de diseño para el riego por gravedad con surcos cerrados. Ingeniería, Investigación y Tecnología, Vol. I., No. 1, UNAM, p.38.

Vázquez E. y De la Cruz R. (1998b). Manual del usuario del los programas ejecutables SURCOS-A y SURCOS-C, para el diseño del riego por gravedad con surcos abiertos y cerrados. Instituto de Ingeniería, UNAM, México.

\section{Apéndice. Simulaciones numéricas adicionales}

La tabla A.1 muestra los resultados de las 40 simulaciones numéricas que complementan los desniveles y eficiencias de la figura 1 del texto para una pendiente. Los datos de K, $\lambda$, L, D y Zm son los mismos de la tabla 2 del texto, según la identificación del surco en la primera columna; la única modificación corresponde a la pendiente $S_{0}$ y con ella, el desnivel $\Delta Z$ que se anotan en la segunda y tercera columnas, respectivamente. En la tabla A.2 se presentan los resultados para dos pendientes; dado que el desnivel $\Delta Z$ toma el mismo valor que los correspondientes a la tabla anterior, no se incluye; pero sí las pendientes $S_{1}$ y $S_{2}$.

Tabla A.1. Resultados de las simulaciones numéricas con una pendiente

\begin{tabular}{lcccccc}
\hline \hline Surco & $\begin{array}{c}\mathrm{S}_{0} \\
(\%)\end{array}$ & $\begin{array}{c}\Delta Z \\
(\mathrm{~cm})\end{array}$ & $\begin{array}{c}\mathrm{Q} \\
(1 / \mathrm{s})\end{array}$ & $\begin{array}{c}\mathrm{ta} \\
(\mathrm{min})\end{array}$ & $\begin{array}{c}\mathrm{Tr} \\
(\mathrm{min})\end{array}$ & $\begin{array}{c}\eta \\
(\%)\end{array}$ \\
\hline \hline Sc (1) & 0.2700 & 62.10 & 0.75 & 324 & 890 & 78 \\
$\mathrm{Cd}(2)$ & 0.2775 & 62.44 & 0.75 & 376 & 830 & 80 \\
Cy $(3)$ & 0.2850 & 62.70 & 0.80 & 280 & 745 & 79 \\
Sc (4) & 0.2925 & 62.89 & 0.80 & 261 & 725 & 77 \\
Cd (5) & 0.3000 & 63.00 & 0.80 & 299 & 675 & 79 \\
Cy (6) & 0.2500 & 63.04 & 0.85 & 239 & 595 & 79 \\
\hline \hline
\end{tabular}


DOI: http://dx.doi.org/10.22201/fi.25940732e.2000.01n3.014

Tabla A.1. Resultados de las simulaciones numéricas con una pendiente (continuación)

\begin{tabular}{|c|c|c|c|c|c|c|}
\hline Surco & $\begin{array}{c}\mathrm{S}_{0} \\
(\%) \\
\end{array}$ & $\begin{array}{c}\Delta \mathrm{Z} \\
(\mathrm{cm}) \\
\end{array}$ & $\begin{array}{c}\mathrm{Q} \\
(1 / S) \\
\end{array}$ & $\begin{array}{c}\mathrm{ta} \\
(\mathrm{min})\end{array}$ & $\begin{array}{c}\mathrm{Tr} \\
(\mathrm{min})\end{array}$ & $\begin{array}{c}\eta \\
(\%) \\
\end{array}$ \\
\hline Sc (7) & 0.3150 & 63.00 & 0.85 & 193 & 583 & 76 \\
\hline $\mathrm{Cd}(8)$ & 0.3225 & 62.89 & 0.80 & 238 & 575 & 77 \\
\hline Cy (9) & 0.2500 & 62.70 & 0.85 & 186 & 500 & 79 \\
\hline $\mathrm{Sc}(10)$ & 0.3375 & 62.44 & 0.80 & 181 & 515 & 76 \\
\hline $\mathrm{Cd}(11)$ & 0.3450 & 62.10 & 0.75 & 223 & 510 & 77 \\
\hline $\mathrm{Cy}_{y}(12)$ & 0.3000 & 61.69 & 0.80 & 158 & 444 & 78 \\
\hline $\mathrm{Sc}(13)$ & 0.3600 & 61.20 & 0.75 & 158 & 460 & 75 \\
\hline $\mathrm{Cd}(14)$ & 0.3675 & 60.64 & 0.70 & 200 & 455 & 76 \\
\hline Cy (15) & 0.3750 & 60.00 & 0.70 & 141 & 415 & 76 \\
\hline $\operatorname{Sc}(16)$ & 0.3825 & 59.29 & 0.70 & 137 & 400 & 74 \\
\hline $\mathrm{Cd}(17)$ & 0.3900 & 58.50 & 0.70 & 168 & 370 & 73 \\
\hline Cy (18) & 0.4000 & 58.00 & 0.70 & 140 & 340 & 73 \\
\hline Sc (19) & 0.2000 & 28.00 & 1.00 & 45 & 200 & 80 \\
\hline $\mathrm{Cd}(20)$ & 0.2100 & 28.35 & 1.00 & 51 & 175 & 82 \\
\hline Cy (21) & 0.2175 & 28.28 & 1.00 & 50 & 162 & 81 \\
\hline $\operatorname{Sc}(22)$ & 0.2250 & 28.13 & 1.00 & 42 & 145 & 81 \\
\hline $\mathrm{Cd}(23)$ & 0.2325 & 27.90 & 0.90 & 52 & 142 & 83 \\
\hline Cy (24) & 0.2400 & 27.60 & 0.90 & 37 & 135 & 79 \\
\hline $\operatorname{Sc}(25)$ & 0.2475 & 27.23 & 0.80 & 38 & 131 & 80 \\
\hline $\mathrm{Cd}(26)$ & 0.2550 & 26.78 & 0.70 & 50 & 130 & 82 \\
\hline Cy (27) & 0.2625 & 26.25 & 0.80 & 40 & 100 & 79 \\
\hline $\mathrm{Sc}(1)$ & 0.3375 & 77.63 & 0.60 & 491 & 1132 & 77 \\
\hline $\mathrm{Sc}(16)$ & 0.2475 & 38.36 & 0.95 & 72 & 272 & 79 \\
\hline $\mathrm{Cd}(17)$ & 0.2550 & 38.25 & 0.95 & 80 & 245 & 81 \\
\hline Cy (18) & 0.2625 & 38.06 & 0.95 & 69 & 230 & 79 \\
\hline Sc (19) & 0.2700 & 37.8 & 0.80 & 70 & 250 & 79 \\
\hline $\mathrm{Cd}(20)$ & 0.2775 & 37.46 & 0.80 & 87 & 224 & 81 \\
\hline Cy (21) & 0.2850 & 37.05 & 0.90 & 59 & 185 & 78 \\
\hline Sc (22) & 0.2925 & 36.56 & 0.80 & 68 & 187 & 79 \\
\hline $\mathrm{Cd}(23)$ & 0.3000 & 36.00 & 0.75 & 83 & 182 & 78 \\
\hline Cy (24) & 0.2500 & 35.36 & 0.70 & 71 & 176 & 77 \\
\hline Sc (25) & 0.3150 & 34.65 & 0.65 & 66 & 167 & 77 \\
\hline $\mathrm{Cd}(26)$ & 0.3225 & 33.86 & 0.70 & 44 & 131 & 80 \\
\hline Cy (27) & 0.2500 & 33.00 & 0.70 & 54 & 120 & 75 \\
\hline
\end{tabular}

Tabla A.2. Resultados de las simulaciones numéricas con dos pendientes

\begin{tabular}{cccccccc}
\hline Surco & $\begin{array}{c}\mathrm{S}_{1} \\
(\%)\end{array}$ & $\begin{array}{c}\mathrm{S}_{2} \\
(\%)\end{array}$ & $\begin{array}{c}\mathrm{Q} \\
(\mathrm{L} / \mathrm{s})\end{array}$ & $\begin{array}{c}\mathrm{ta} \\
(\mathrm{min})\end{array}$ & $\begin{array}{c}\mathrm{Tr} \\
(\mathrm{min})\end{array}$ & $\begin{array}{c}\eta_{1} \\
(\%)\end{array}$ & $\begin{array}{c}\mathrm{Ag} \\
(\%)\end{array}$ \\
\hline $\mathrm{Sc}(1)$ & 0.2667 & 0.1333 & 0.75 & 325 & 832 & 84 & 7.1 \\
$\mathrm{Cd}(2)$ & 0.2800 & 0.1400 & 0.75 & 376 & 773 & 86 & 7.0 \\
$\mathrm{Cy}(3)$ & 0.2900 & 0.1450 & 0.80 & 281 & 702 & 84 & 6.0 \\
$\mathrm{Sc}(4)$ & 0.3000 & 0.1500 & 0.80 & 262 & 663 & 85 & 9.4 \\
$\mathrm{Cd}(5)$ & 0.3100 & 0.1550 & 0.80 & 299 & 625 & 84 & 6.0 \\
$\mathrm{Cy}(6)$ & 0.3200 & 0.1600 & 0.85 & 234 & 565 & 83 & 4.8 \\
\hline
\end{tabular}


DOI: http://dx.doi.org/10.22201/fi.25940732e.2000.01n3.014

Tabla A.2. Resultados de las simulaciones numéricas con dos pendientes (continuación)

\begin{tabular}{|c|c|c|c|c|c|c|c|}
\hline Surco & $\begin{array}{l}S_{1} \\
(\%)\end{array}$ & $\begin{array}{c}\mathrm{S}_{2} \\
(\%)\end{array}$ & $\begin{array}{c}\mathrm{Q} \\
(1 / \mathrm{s})\end{array}$ & $\begin{array}{c}\mathrm{ta} \\
(\mathrm{min})\end{array}$ & $\begin{array}{c}\mathrm{Tr} \\
(\mathrm{min})\end{array}$ & $\begin{array}{r}\eta_{1} \\
(\%)\end{array}$ & $\begin{array}{l}\mathrm{Ag} \\
(\%)\end{array}$ \\
\hline Sc (7) & 0.3300 & 0.1650 & 0.85 & 194 & 530 & 84 & 9.5 \\
\hline $\mathrm{Cd}(8)$ & 0.3400 & 0.1700 & 0.80 & 238 & 530 & 84 & 8.3 \\
\hline Cy (9) & 0.3500 & 0.1750 & 0.85 & 180 & 478 & 83 & 4.8 \\
\hline Sc (10) & 0.3600 & 0.1800 & 0.80 & 182 & 475 & 83 & 8.4 \\
\hline $\mathrm{Cd}(11)$ & 0.3700 & 0.1850 & 0.75 & 223 & 477 & 83 & 7.2 \\
\hline Cy (12) & 0.3800 & 0.1900 & 0.80 & 155 & 418 & 83 & 6.0 \\
\hline Sc (13) & 0.3900 & 0.1950 & 0.75 & 158 & 414 & 83 & 9.6 \\
\hline $\mathrm{Cd}(14)$ & 0.4000 & 0.2000 & 0.70 & 201 & 420 & 82 & 7.3 \\
\hline Cy (15) & 0.4100 & 0.2050 & 0.70 & 142 & 387 & 82 & 7.3 \\
\hline Sc (16) & 0.4200 & 0.2100 & 0.70 & 138 & 356 & 83 & 10.8 \\
\hline $\mathrm{Cd}(17)$ & 0.4300 & 0.2150 & 0.70 & 169 & 335 & 81 & 9.9 \\
\hline Cy (18) & 0.4400 & 0.2200 & 0.70 & 141 & 311 & 81 & 9.9 \\
\hline $\mathrm{Sc}(19)$ & 0.4500 & 0.2250 & 1.00 & 45 & 190 & 83 & 3.6 \\
\hline $\mathrm{Cd}(20)$ & 0.4600 & 0.2300 & 1.00 & 51 & 167 & 86 & 4.7 \\
\hline Cy (21) & 0.4700 & 0.2350 & 1.00 & 51 & 150 & 87 & 6.7 \\
\hline $\mathrm{Sc}(22)$ & 0.4800 & 0.2400 & 1.00 & 42 & 135 & 87 & 6.7 \\
\hline $\mathrm{Cd}(23)$ & 0.4900 & 0.2450 & 0.90 & 53 & 130 & 91 & 8.8 \\
\hline Cy (24) & 0.5000 & 0.2500 & 0.90 & 37 & 122 & 85 & 7.1 \\
\hline $\mathrm{Sc}(25)$ & 0.5100 & 0.2550 & 0.80 & 39 & 118 & 88 & 9.1 \\
\hline $\mathrm{Cd}(26)$ & 0.5200 & 0.2600 & 0.70 & 50 & 116 & 90 & 8.9 \\
\hline Cy (27) & 0.5333 & 0.2667 & 0.80 & 40 & 90 & 88 & 10.2 \\
\hline $\mathrm{Sc}(1)$ & 0.4500 & 0.2250 & 0.60 & 500 & 1072 & 82 & 6.1 \\
\hline $\mathrm{Sc}(16)$ & 0.3300 & 0.1650 & 0.95 & 73 & 257 & 84 & 6.0 \\
\hline $\mathrm{Cd}(17)$ & 0.3400 & 0.1700 & 0.95 & 80 & 225 & 88 & 8.0 \\
\hline Сy (18) & 0.3500 & 0.1750 & 0.95 & 70 & 212 & 86 & 8.1 \\
\hline $\mathrm{Sc}(19)$ & 0.3600 & 0.1800 & 0.80 & 71 & 230 & 86 & 8.1 \\
\hline $\mathrm{Cd}(20)$ & 0.3700 & 0.1850 & 0.80 & 88 & 204 & 89 & 9.0 \\
\hline Cy (21) & 0.3800 & 0.1900 & 0.90 & 59 & 167 & 87 & 10.3 \\
\hline $\mathrm{Sc}(22)$ & 0.3900 & 0.1950 & 0.80 & 68 & 168 & 88 & 10.2 \\
\hline $\mathrm{Cd}(23)$ & 0.4000 & 0.2000 & 0.75 & 83 & 166 & 85 & 8.2 \\
\hline Cy (24) & 0.4100 & 0.2050 & 0.70 & 71 & 160 & 85 & 9.4 \\
\hline $\operatorname{Sc}(25)$ & 0.4200 & 0.2100 & 0.65 & 66 & 150 & 86 & 10.5 \\
\hline $\mathrm{Cd}(26)$ & 0.4300 & 0.2150 & 0.70 & 44 & 119 & 87 & 8.0 \\
\hline $\mathrm{Cy}(27)$ & 0.4400 & 0.2200 & 0.70 & 55 & 108 & 83 & 9.6 \\
\hline
\end{tabular}

\section{Semblanza del autor}

Ernesto Vázquez-Fernández. Egresado de la UNAM, ha sido profesor de la Universidad Juárez Autónoma de Tabasco y de la DEPFI, UNAM. Asimismo, se ha desempeñado como especialista en ingeniería hidráulica en los sectores público y privado. Actualmente, labora en el Instituto de Ingeniería, UNAM. 